\title{
Adapalene in management of molluscum contagiosum in pediatric population
}

\section{Mrinal Gupta}

\author{
Department of Dermatology, Treatwell Skin Centre, Jammu and Kashmir, India
}

Corresponding author: Dr. Mrinal Gupta, E-mail: drmrinalgupta@yahoo.com

Sir,

Molluscum contagiosum (MC) is a common viral infection caused by DNA Poxvirus. It has no other reservoir than humans and is transmitted directly by skin-to-skin contact or indirectly through fomites. It's a widely prevalent infection commonly affecting children, sexually active adults, and immunodeficient individuals [1]. A large number of treatments exist for treatment of $\mathrm{MC}$ which include destructive therapies like curettage, cryotherapy, pricking with a sterile needle, electrodesiccation, photodynamic therapy and lasers. Other topical medications include salicylic acid, tretinoin, potassium hydroxide, trichloroacetic acid and imiquimod. However, no therapy is universally effective and most of these treatment modalities are not well tolerated by children [2-4].

In this study, we tried to evaluate the safety and efficacy of Adapalene $0.1 \%$ gel in MC in the pediatric age group.

It was a prospective study carried out over a period of one year in our centre where children aged $3-18$ years with molluscum contagiosum were treated with bed time application of adapalene $0.1 \%$ and were followed up for three months to evaluate the efficacy and any adverse effects. Those patients who were on any systemic therapy in the past 8 weeks were excluded from the study. All other topical treatments were stopped prior to the study. The effect of treatment was evaluated monthly for three months.

A total of 25 children aged between $3-18$ years with a mean age $7.14 \pm 1.36$ years (M: F 14:11) were included in the study and were followed up over a period of three months. Out of 25 patients, 20 patients completed the study. The total number of molluscum per patient varied from 1-24 with a mean of 3.4 lesions per patient and the mean duration of lesions was 9 weeks \pm 2.36 weeks. After one month of treatment, two patients had complete resolution of lesions, whereas after two months, five patients had complete clearance and four had partial response. At the end of three months of follow up, eleven patients (55\%) showed complete clearance of lesions, three patients $(15 \%)$ had partial resolution of lesions where a few molluscum were still present, whereas six patients $(30 \%)$ had no response. Side effects of adapalene were also observed and over the course of study, five patients $(25 \%)$ showed some adverse effects like dryness $(n=1)$, erythema $(n=3)$ or irritation $(n=4)$.

A large variety of treatment modalities have been used for molluscum but most of these involve destructive modalities which are not favored in pediatric population. The ideal agent for children should be effective, less irritant, well tolerated and easily available, which can be conveniently applied. Adapalene is a retinoid with ability to alter keratinization and affinity for retinoic acid receptor (RARY). It has been found to have anti-inflammatory action, inhibits cell proliferation, and modulates cell differentiation. A few studies have demonstrated the efficacy and safety of adapalene $0.1 \%$ gel in the management of molluscum in children [5].

Kashif et al studied the efficacy and safety of trichloroacetic acid $35 \%$ versus adapalene $0.1 \%$ in treatment of molluscum contagiosum in children, in which they treated 30 children each with trichloroacetic acid and adapalene and observed the clinical response over a period of six weeks. They observed that complete clinical clearance was seen in observed in 25 (83.3\%)

\footnotetext{
How to cite this article: Gupta M. Adapalene in management of molluscum contagiosum in pediatric population. Our Dermatol Online. 2019;10(4):391-392.

Submission: 20.05.2018; Acceptance: 25.09.2018

DOI:10.7241/ourd.20194.22
} 
of patients treated with TCA and $20(66.7 \%)$ patients treated with adapalene, however, adverse effects were more common in TCA group with $30 \%$ patients $(n=9)$ having adverse effects like erythema, scarring and dyspigmentation as compared to $3.3 \%(\mathrm{n}=1)$ patients in adapalene group [5]. In our study, we observed a complete clearance in $55 \%$ and adverse effects were observed in $25 \%$ patients.

Treatment options like extirpation, electrodessication, cryosurgery, TCA and KOH application are the commonly used techniques but they can cause irritation and pain and can lead to scarring, so their use in children is not universally accepted. Adapalene can be used a safer alternative to other modalities in the treatment of MC especially in children.

This study has certain limitations. First of all, it was a single centered trial conducted in a limited population size and the dropout rate was quite high. Moreover, application of adapalene was not carried out under medical supervision as the patients were sent home and had to apply it by themselves.

\section{CONSENT}

The examination of the patient was conducted according to the Declaration of Helsinki principles.

\section{REFERENCES}

1. Hanson D, Diven DG. Molluscum contagiosum. Dermatol Online J. 2003;9:2.

2. Metkar A, Pande S, Khopkar U. An open, nonrandomized, comparative study of imiquimod $5 \%$ cream versus $10 \%$ potassium hydroxide solution in the treatment of molluscum contagiosum. Indian J Dermatol VenereolLeprol. 2008;74:614-8.

3. Bayerl C, Feller G, Goerdt S. Experience in treating molluscum contagiosum in children with imiquimod 5\% cream. Br J Dermatol. 2003;149:25-9.

4. Mahajan BB, Pall A, Gupta RR. Topical 20\% KOH-An effective therapeutic modality for molluscum contagiosum in children. Indian J Dermatol VenereolLeprol. 2003;69:175-7.

5. Kashif M, Tahir R, Hussain I. Efficacy and safety of trichloroacetic acid $35 \%$ versus adapalene $0.1 \%$ in treatment of molluscum contagiosum in children. J Pak Associat Dermatol. 2017;26:366-70.

Copyright by Mrinal Gupta. This is an open-access article distributed under the terms of the Creative Commons Attribution License, which permits unrestricted use, distribution, and reproduction in any medium, provided the original author and source are credited.

Source of Support: Nil, Conflict of Interest: None declared. 